Rev Biomed 2003; 14:269-278.

\title{
Género, violencia y la epidemia del VIH/Sida en Yucatán, México.
}

Revisión

Ligia Vera-Gamboa.

Centro de Investigaciones Regionales "Dr. Hideyo Noguchi", Universidad Autónoma de Yucatán, Mérida, Yucatán, México.

\section{RESUMEN.}

El síndrome de inmunodeficiencia humana(Sida) se considera una de las más devastadoras enfermedades que la humanidad haya enfrentado. Las infecciones en mujeres han ido en aumento, representando para finales de 2001 la mitad de las infecciones, en comparación con el 25\% que representaban en 1992. En Yucatán, durante 2001, se documentaron 26 casos femeninos, lo que representa cerca del $18 \%$ de todas las mujeres mayores de 15 años infectadas desde 1988 en el estado de Yucatán, México.

En el presente trabajo se revisan los conceptos en torno a la relación que existe entre el género, la violencia y la epidemia del Sida y la vulnerabilidad femenina a la infección por el virus de la inmunodeficiencia humana (VIH) en Yucatán, México.

Se hace notar la vulnerabilidad femenina a través de patrones sociales aprendidos culturalmente, incluídos los comportamientos sexuales, la repercusión de la violencia y como éstos afectan la prevención del VIH/Sida en el caso de las mujeres. Por último, se proponen alguna líneas de acción para reducir la vulnerabilidad femenina a la epidemia del VIH/Sida. (Rev Biomed 2003; 14:269-278)

Palabras clave: VIH, Sida, sexualidad, género, violencia de género.

\section{SUMMARY.}

\section{Gender, violence and the HIV/AIDS epidemic} in Yucatan, Mexico.

HIV/Aids is considered one of the most destructive disease that humanity has faced. Infections in women have increased, representing half of the infections at the end of 2001 compared with $25 \%$ in 1992. Twenty six female cases were reported in Yucatan in 2001, which represented approximately $18 \%$ of women over 15 years of age infected since the first female case in the state of Yucatan in 1988. In this work concepts are reviewed with regard to the relation between sexuality, violence, and the HIV/ Aids epidemic, and female vulnerability to HIV

Solicitud de sobretiros: M.C. Ligia Vera-Gamboa. Centro de Investigaciones Regionales "Dr. Hideyo Noguchi", Universidad Autónoma de Yucatán, Av. Itzáes No. 490 x 59, C.P. 97000, Mérida, Yucatán, México. Correo electrónico: vgamboa@tunku.uady.mx 


\section{Vera-Gamboa.}

infection in Yucatan, Mexico.

Female vulnerability is noted daily through culturally learnt social patterns, including sexual behavior and the repercussion of violence, and these affect the prevention of HIV/Aids among women. Finally some courses of action are proposed to reduce female vulnerability to the HIV/Aids epidemic. (Rev Biomed 2003; 14:269-278)

Key words: HIV, Aids, sexuality, sex, sexual violence.

\section{INTRODUCCION.}

En el verano de 1981, la humanidad conoció de una enfermedad hasta entonces no descrita, el Síndrome de Inmunodeficiencia Adquirida (Sida)(1,2). Hoy es considerada una de las más devastadoras enfermedades que la humanidad haya enfrentado, como lo demuestran, entre otros hechos, que a poco más de 20 años de su aparición, el Sida ocupe hoy la cuarta causa de muerte a nivel mundial. A fines de 2001, se reportó que 40 millones de personas vivían con el VIH/Sida y también se reportaron 3 millones de defunciones (3), que se sumaban a los cerca de 22 millones reportados hasta el año 2000 (4), haciendo un total de 65 millones afectadas, considerando las muertes ocurridas y las personas que viven con el VIH.

A finales de 2001, el Programa Conjunto de las Naciones Unidas sobre el VIH/Sida (ONUSIDA) reportó que en ese año ocurrieron cinco millones de nuevas infecciones por el VIH/Sida, que representan un incremento mayor del $10 \%$ de los casos reportados en 2000 (3). La mayoría de las nuevas infecciones ocurren en adultos jóvenes y son particularmente vulnerables las mujeres jóvenes. De las infecciones registradas para 2001, dos millones correspondieron a mujeres, con lo que la cifra de mujeres afectadas por el VIH/Sida totalizó 17.6 millones en el mundo, representando cerca del $50 \%$ de todos los adultos afectados por el VIH/Sida (3). Las defunciones de mujeres han rebasado los diez millones, más de tres veces las estimaciones que en
1990 hiciera la Organización Mundial de la Salud para el año dos mil, lo que representa la pérdida de vidas jóvenes y en plena productividad. Asimismo, hay que señalar que de las 14,000 infecciones que ocurren cada día, $95 \%$ corresponden a países en desarrollo, como es México, y de los casos de 15 a 49 años (12 000), la mitad son mujeres, cifra superior al 25\% que representaban en 1992 (5).

A Junio de 2002, el estado de Yucatán ocupaba el sexto lugar nacional (6) por tasa de incidencia y, al igual que en todo el mundo, los casos en mujeres han ido en aumento. Sólo en el año 2001 los Servicios de Salud de Yucatán, documentaron 26 nuevos casos femeninos (7), cifra que representa el $18 \%$ (26/148) del total de las mujeres mayores de 15 años con diagnóstico de Sida en el estado de Yucatán, desde el reporte del primer caso femenino en el estado, en 1988.

\section{GENERO Y VULNERABILIDAD FEMENINA AL VIH/SIDA.}

Es importante señalar lo que se entiende por este término. El género como un concepto, no se refiere únicamente a los hombres y las mujeres sino también a las relaciones entre ellos y a la construcción social que se hace de dichas relaciones. En este amplio sentido, el género se convierte en un principio organizacional de la sociedad(8).

El género determina lo que se espera de hombres y mujeres, incluidos sus comportamientos sexuales. Por lo general, los varones son socializados fuera del hogar con el apoyo de sus pares en la calle o fuera de casa. En contraste, las mujeres son socializadas en el hogar. Como resultado de esto, las mujeres están a menudo poco informadas sobre la sexualidad, el sexo se convierte en la mayoría de las veces en sinónimo de reproducción y es así como generalmente la maternidad se convierte en el principal referente de identidad femenina. En tanto, en los hombres, lo masculino se reconoce como un proceso biológico, natural, "incontrolable" y necesario de ejercer para demostrar la hombría.

En cuanto a la epidemia del VIH/Sida se conoce que las mujeres son más vulnerables biológicamente

\section{Revista Biomédica}


a la infección que los hombres (9). Existe de 2 a 12 veces mayor probabilidad de que ocurra más fácilmente la trasmisión de hombre a mujer que visceversa (10), en especial si se trata de mujeres adolescentes, en quienes el epitelio vaginal es aún inmaduro $(11,12)$. Epidemiológicamente también existe mayor vulnerabilidad (9), ya que es un hecho conocido que los hombres habitualmente tienen más parejas que las mujeres, ya que éstas se casan, por lo general, con hombres mayores, quienes ya han tenido una o más parejas (13). Hay que señalar que la mujer también se casa con la historia sexual de su pareja.

Desde el punto de vista social, la vulnerabilidad femenina $(8,10)$ también es mayor que la de los hombres. La vulnerabilidad social se entiende como la suma de factores socioculturales, económicos y políticos que limitan las opciones individuales de reducción de riesgo en general. En otras palabras, la vulnerabilidad es un indicador de inequidad que afecta de manera más directa a las mujeres. La vulnerabilidad social incluye la discriminación, estigmatización y la marginación de ciertos grupos. En el caso de las mujeres, el analfabetismo y en general la dificultad o falta de acceso a servicios básicos, como son educación, empleo y salud, incrementa esta vulnerabilidad.

Las normas de género interfieren en los conocimientos y prevención del VIH en hombres y mujeres, lo que unido a las actitudes y comportamientos conforman el riesgo individual a la infección por VIH. Estas normas y representaciones llevan a establecer estereotipos y patrones de moral sexual desiguales para los hombres y las mujeres, determinando así comportamientos diferentes de exposición a la infección por VIH, lo que favorece el riesgo de infección por VIH en la mujer. Inclusive, no todas las mujeres tienen el mismo grado de riesgo (14).

Un ejemplo de esto, es el alto valor que la cultura mexicana confiere a la virginidad, hecho que en no pocas ocasiones lleva a las mujeres a la realización de prácticas sexuales de mayor riesgo, como son las relaciones anales sin protección, con la finalidad de conservar la virginidad y evitar el embarazo, o bien, para complacer a la pareja (15), sin percibir que esta práctica realizada sin protección, es la de mayor riesgo para el VIH/Sida, en caso de que el compañero esté infectado. Como éste, otros comportamientos de riesgo se vuelven más probables. Así, por ejemplo, muchas mujeres pueden sospechar que su compañero mantiene relaciones extrapareja, y son incapaces de negociar protección para ellas durante la práctica sexual consensual, por temor a ser abandonadas y dejar a sus hijos en el desamparo. O bien, porque las mujeres, por lo general, han sido socializadas para dar placer como una deferencia a los hombres, olvidando su propio placer y erotismo. De hecho, en algunos países es una práctica culturalmente aceptada la infidelidad de los hombres casados (16), por lo que las relaciones sexuales y la violencia sexual contra las mujeres, fenómenos en crecimiento, se relacionan con el género y con las consecuencias inherentes para la prevención del VIH/Sida (16-18).

En Yucatán, los casos femeninos registrados por los Servicios de Salud de Yucatán, reportan que las mujeres afectadas son en su gran mayoría amas de casa, mujeres sin aparentes factores de riesgo. Sin embargo, en un trabajo realizado en el Centro de Investigaciones Regionales “Dr. Hideyo Noguchi”'de la Universidad Autónoma de Yucatán (CIR) (19), entre mujeres embarazadas sin acceso a seguridad social, encontramos que el $10 \%$ de poco más de 300 mujeres, no aceptó realizarse la prueba de anticuerpos contra el VIH, ofrecida bajo los lineamientos establecidos en la Norma Oficial Mexicana 010-SSA2-1993, aduciendo que para ello requerían permiso de su pareja, o bien, por no considerarse en riesgo al mantener relaciones sexuales sólo con su pareja. De aquellas que aceptaron, la principal razón para realizársela fue conocer el estado de su salud en función de su bebé. Estos datos coinciden con lo reportado previamente por Pedrosa (20) y refuerzan el hecho de que la mujer antepone la salud de los demás miembros de la familia a la propia. Además, este estudio nos permitió identificar que en el contexto de estas mujeres, estar 


\section{Vera-Gamboa.}

casadas las hace considerarse inmunes a la infección por VIH/Sida y que poseen un escaso conocimiento sobre el VIH/Sida, dato similar a lo reportado por Goldzieher (16).

Al Sida se le conoce también como la enfermedad de la pobreza. Este hecho, expone especialmente a las mujeres a la infección por el VIH. Así habitualmente desempeñan una doble o triple jornada de trabajo, lo que unido a una deficiente alimentación, falta de descanso, estrés emocional y/ o físico al interior de la familia, incluyendo las relaciones forzadas desprotegidas, las hacen más vulnerables a esa infección.

En Yucatán, la mujer fue infectada en los inicios de la epidemia por transfusiones sanguíneas $(10,13)$. Actualmente, la vía sexual ha ido ganando terreno y el 80\% los casos de Sida en mujeres mayores de 15 años, son mujeres amas de casa, cuyo factor de riesgo fue la práctica sexual sin protección con su pareja estable infectada $(21,22)$. La experiencia obtenida durante la epidemia del VIH/Sida en Yucatán, señala que cuando una mujer conoce que su pareja está infectada, lo cuida y cuando fallece se hace cargo de la familia, sin considerar en cómo se infectó, hecho también reportado por Liguori (10). Si la mujer es quien está infectada, por lo general es abandonada, a pesar de que dicha infección la haya adquirido de su propia pareja. Esta situación se presenta incluso en las trabajadoras sexuales, quienes se protegen con sus clientes, más no así con su pareja habitual (10). Una consecuencia de la infección en mujeres jóvenes y en edad reproductiva, es el incremento de casos de menores infectados por la vía del embarazo o la lactancia. Muchas veces, el diagnóstico en un hijo ha permitido conocer que la madre también está infectada. Una carga emocional en estas mujeres, es la enorme preocupación de dejar huérfanos a sus hijos.

La infección por VIH/Sida hace a la mujer más vulnerable a la probabilidad de desarrollar cáncer cervicouterino, una de las primeras causas de muerte en el estado de Yucatán en mujeres jóvenes (23). En un trabajo previo realizado en Yucatán en mujeres con infección por VIH, también encontramos un mayor riesgo de desarrollar displasia cervical, lesión precursora del cáncer cervicouterino (24).

\section{VIOLENCIA Y VIH/SIDA.}

De conformidad con lo establecido en la "Declaración para la Erradicación de Todas las Formas de Discriminación Contra la Mujer" (25), la violencia sexual es un abuso basado en el género y realizado la mayoría de las veces por un varón en el que la mujer confía.

La violación y la violencia familiar son causas de discapacidad y muerte en mujeres en edad reproductiva, independientemente de se viva en un país desarrollado o en vías de desarrollo. Las consecuencias de la violencia de género representan cerca de un año de vida perdido en el grupo etáreo de 15 a 44 años, por cada cinco años de vida saludable (26). Es así como recientemente la violencia ha sido señalada como causa significativa de enfermedades y muertes femeninas, al margen de que ocurran como producto de violencia física, durante el período de gestación o como resultado de acciones coersitivas de tipo sexual o a través de abuso sexual infantil. Cada día existe mayor evidencia de asociación entre violencia y trastornos de salud física y mental (27). Aun cuando no existen datos suficientes acerca del problema, algunos estudios arrojan datos interesantes. Un estudio de la OMS (1997) en una selección de encuestas de población, mostró que entre 20 y $50 \%$ de las mujeres encuestadas, dijeron haber sido objeto de violencia física por parte de un compañero masculino íntimo, al menos una vez en su vida, y una de cada 5 mujeres había sido forzada a tener relaciones sexuales en contra de su voluntad (28).

A nivel familiar, el dominio y control masculino sobre la riqueza son importantes, ya que en lo social se traducen en títulos de propiedad del hombre sobre la mujer, como si ésta fuera un objeto. La masculinidad vinculada a la agresión y dominio, y la aceptación de la violencia interpersonal para resolver conflictos, convierten a la violencia en uno de los elementos de subordinación y cuando la mujer se atreve a transgredir las atribuciones impuestas de

\section{Revista Biomédica}




\section{Género, violencia y VIH/Sida.}

manera tradicional por la sociedad, la violencia se hace mucho más frecuente.

Finkler (29), en un estudio realizado en México en 1997, señala que las ideologías tradicionales refuerzan la dependencia económica de la mujer sus conceptos sobre su papel en el hogar y su inferioridad social y la forma en que éstas se refuerzan inclusive a través de la práctica médica. Señala que el poder que estas ideologías conceden al hombre y el uso de la violencia física generan "lesiones de vida", las cuales se asocian a enfermedad y se convierten en causa de cuadros subagudos, no amenazantes para la vida pero sí difíciles de manejar en los servicios de salud. Las normas culturales que rodean a la violencia se extienden mas allá de lo individual, abarcan a la familia, la comunidad y la sociedad en su conjunto. En no pocos ambientes, se considera la violencia como normal. A los niños se los socializa para ser agresivos y sexualmente activos, pero a las niñas se les enseña a resistir la actividad sexual y ser dulces y tiernas.

Así, la violencia no sólo causa daños físicos y psicológicos a las mujeres, sino también implica riesgos para sus hijos. Presenciar la violencia conyugal incrementa en los niños las probabilidades de sufrir depresión, ansiedad, trastornos de conducta y retrasos en su desarrollo cognoscitivo. Además, aumenta a su vez el riesgo de convertirse en víctima de maltrato o en hombres y mujeres violentos (26, 30-32). Los hijos de mujeres violentadas, sean víctimas de la misma o sólo testigos de ella, presentan problemas de conducta, escolares y de relaciones interpersonales con sus compañeros. Varios investigadores $(17,30-32)$ han referido que cuando un niño es testigo frecuente de violencia materna presenta asociación con "conductas de alto riesgo", como la práctica de sexo no seguro en etapas posteriores de la vida. Igualmente, ser testigo de violencia doméstica contribuye a la violencia general, en el sentido de que estos niños identifican la violencia como medio de resolución de conflictos, además de reforzar los estereotipos y las desigualdades de género.

Una investigación realizada en 16 delegaciones del Distrito Federal, México, reveló que el 61.5\% de las mujeres dedicadas exclusivamente a labores domésticas habían sido maltratadas físicamente alguna vez por sus maridos o concubinos (33). Igualmente, una encuesta realizada en hospitales de urgencia de esa misma ciudad, revisó casos que presentaron lesiones traumáticas y tuvieron como causa hechos violentos; estos representaron $14 \%$ del total de la demanda de atención médica. En el $95 \%$ de ellos, la víctima fue del sexo femenino y la producción de lesiones se asociaba a intoxicación alcohólica de parte del agresor (34).

De los escasos estudios que existen en México en relación a la violencia sexual, Ramos y col. (35) encontraron que de 345 usuarias de un centro de salud de la ciudad de México D.F. y cuyas edades fluctuaron de 10 a 65 años, una de cada cinco había experimentado algún tipo de violencia sexual de su pareja y encontraron una asociación significativa entre algunas formas de violencia sexual y depresión, ideación e intento suicida y el uso de psicofármacos.

En nuestro medio, existe escasa información sobre la violencia familiar. Un estudio realizado por Oliva (36) sobre violencia doméstica en usuarias de una clínica de planificación familiar en Mérida, Yucatán, reportó que más del 50\% de 76 mujeres entrevistadas reconocieron haber vivido situaciones de violencia. La violencia física estuvo presente en $25 \%$, la violencia psicológica o emocional en $47 \%$ y la violencia sexual en el 18\%. El $31 \%$ de ellas dijeron haber sufrido violencia en todas sus formas y niveles posibles, y de $76 \%$ de quienes perciben a sus parejas como malhumoradas o agresivas, el $46 \%$ atribuyó al alcohol o drogas la intensificación de la violencia. Por lo que respecta a daños a la salud, el 13\% de ellas refirió haber presentado alguna infección de transmisión sexual, embarazos no deseados y alteraciones a su salud mental, como son depresión, irritabilidad, insomnio y ansiedad. Además, el hecho de contar con ingresos propios (46\%) no fue una condición que favoreció el desarrollo de acciones en contra de su o sus agresores, haciéndose presente la influencia sociocultural de género.

Estos datos concuerdan con lo señalado en la

Vol.14/No.4/Octubre-Diciembre, 2003 


\section{Vera-Gamboa.}

literatura mundial $(25,26)$ que señala que la violencia doméstica, en especial la violencia sexual, daña la salud reproductiva de la mujer directamente al causar embarazos no deseados, infecciones de transmisión sexual, incluyendo el VIH/Sida, e indirectamente al causar daño emocional y comportamientos que las llevan a una variedad de conductas sexuales arriesgadas.

Estos resultados coinciden con los conceptos de García Ramírez (37): "en la violencia emocional o psicológica, la fuerza gira en torno al atemorizamiento y a la humillación de la víctima, recalca las diferencias jerárquicas entre los géneros y simboliza el sometimiento de la mujer al poder político del hombre”. También coinciden con el sentir de las mujeres en cuanto a la ponderación de daños: "las mujeres consideran que el abuso psicológico y la humillación son más devastadores que la agresión física” (38). En la opinión de Granados (39): "la violencia en el matrimonio y en todas las formas de conyugalidad, existe de manera generalizada y masiva. Ni la sociedad, ni las víctimas, ni los hombres agresores la conciben como tal. Ella es su mujer"

Se ha reconocido que la fidelidad mutua y el uso del condón son medidas efectivas de prevención para el VIH/Sida. Sin embargo, aun cuando la fidelidad mutua puede ser una medida para algunas parejas, no lo es para la mayoría. En cuanto al condón, su uso depende del hombre y la negociación del mismo se relaciona con promiscuidad, infidelidad y prostitución, apreciaciones que pueden provocar una reacción violenta en el compañero, especialmente en una cultura machista como la mexicana. En los niveles socioeconómicos más desprotegidos, la ignorancia se suma a este hecho.

El temor a la violencia también obstaculiza los esfuerzos internacionales y locales para reducir la transmisión de madre a hijo del VIH, temor que lleva a las mujeres a no realizarse la prueba o no acudir por los resultados de la misma. Asimismo, en países como el nuestro, donde la lactancia es una tradición cultural, particularmente en áreas rurales y/o en mujeres indígenas, recomendar a las mujeres VIH positivas el uso del biberón, puede ser condenarlas al aislamiento y discriminación, limitando de esta manera los esfuerzos de disminución de la transmisión perinatal (18).

Un grupo de mujeres doblemente vulnerables, son las mujeres trabajadoras sexuales (MTS). En Yucatán, al igual que en la mayor parte de la República Mexicana, el trabajo sexual no se encuentra reglamentado, considerándose únicamente como una conducta “inmoral". Por esta razón, las MTS son estigmatizadas socialmente por el solo hecho de ejercer esta actividad.

En Yucatán, México, entre 1987 y 1992 la prevalencia reportada de infección del VIH entre MTS fue de $0.4 \%$ (40-41). Estos resultados coinciden con lo reportado en la literatura acerca de la prevalencia de infección por VIH en las MTS de Latinoamérica (42), y en México (42-45). Las mujeres que ejercen el trabajo sexual son por lo general de nivel socioeconómico bajo, baja escolaridad y, al igual que muchas otras mujeres, son sujetos de violencia familiar. En el desempeño de su oficio también son víctimas de abusos sociales. Además, cerca de la mitad de ellas cuentan con pareja estable. Es importante señalar que estas mujeres aun cuando más del $90 \%$ de ellas reconoce ser sujetos de riesgo para la infección por el VIH; sólo el 16\% utiliza el condón en todas sus prácticas sexuales, más del $60 \%$ lo usa de manera irregular e inconsistente con sus clientes y nunca con su pareja habitual (46). Como ya ha sido señalado, en los datos epidemiológicos del CIR, las MTS infectadas, lo han sido principalmente a través de sus parejas bisexuales, más que por el ejercicio mismo del trabajo sexual (22). Aunque estas mujeres, por lo general, son consideradas como el puente potencial que puede diseminar la epidemia entre la población bisexual y heterosexual, debe tenerse en cuenta la mayor eficacia de contagio del hombre a la mujer, como ya ha sido señalado, por lo que, las MTS tienen mayor oportunidad de ser infectadas por sus clientes, con sólo considerar el número de parejas y de prácticas sexuales.

Asimismo, ha sido señalado que dependiendo

\section{Revista Biomédica}


Género, violencia y VIH/Sida.

del nivel de autoestima, la MTS recurrirá o no a medidas preventivas (47). En un estudio realizado en MTS en la ciudad de Mérida, Yucatán, para conocer su nivel de autoestima, se encontró que $65 \%$ de ellas presentaron una baja autoestima, hecho a considerar cuando estas mismas mujeres refieren encontrarse indiferentes o insatisfechas durante la realización de su trabajo (48), lo que sin duda puede estar incidiendo en la falta de adopción de conductas preventivas.

\section{PROPUESTAS.}

¿Hacia donde dirigir las acciones?

1.- Vigilar y defender el respeto a los derechos humanos de las mujeres particularmente en los servicios de salud y laborales.

2.- Es urgente legislar acerca del trabajo doméstico y la corresponsabilidad de la pareja en la convivencia diaria.

3.- Es pertinente sustentar jurídicamente sobre la situación legal y de seguridad económica de la mujer en caso de divorcio, ya que hoy, cuando la mujer se divorcia, queda desprotegida, lo que agrava su condición si no cuenta con un empleo remunerado y con seguridad social.

4.- Es prioritario mejorar las condiciones laborales de las mujeres desde el punto vista legal y social, en la búsqueda de la equidad social y de género.

5.- Elevar el comercio sexual a la calidad de trabajo, tal como ha ocurrido en otros países como Uruguay, por ejemplo, considerando que para que la prostitución exista, hay toda una infraestructura empresarial y social que la permite.

6.- Realizar un foro sobre trabajo sexual y género que permita encontrar acciones de prevención para el VIH/Sida en este grupo vulnerable. La idea es que en él participen el sector salud, la Iglesia, los sectores educativos, organizaciones gubernamentales y no gubernamentales, partidos políticos, el Congreso del Estado de Yucatán y, especialmente, las MTS. El ejemplo en México lo han puesto los estados de Veracruz y Puebla.

7.- El Sector Salud deberá difundir la Norma
Oficial Mexicana de Violencia Familiar; sólo así el personal de salud visualizará la violencia familiar como un problema de salud pública, además de permitir tener un mayor conocimiento de esa entidad y su dinámica epidemiológica en México y en nuestro estado en particular.

8.- Las autoridades gubernamentales deben realizar esfuerzos para que las mujeres tengan mayor acceso a los servicios básicos, como son educación, salud y empleo. En especial para las mujeres de áreas rurales, con la finalidad de disminuir su vulnerabilidad social.

En relación a la prevención del VIH/Sida :

1.- Promover acciones entre el sector privado y gubernamental para realizar campañas de información y prevención específicas, dirigidas a las mujeres, especialmente las amas de casa, hoy por hoy el grupo femenino mayormente afectado.

2.- Es prioritaria la integración efectiva de los programas de salud reproductiva y materno infantil y los programas de VIH/Sida y otras ITS, tal como se ha sugerido internacionalmente, pero sin olvidar la dignidad y el respeto a los derechos de la mujer, observando los lineamientos establecidos en la Norma Oficial Mexicana. Esto evitaría en lo posible, que el diagnóstico de infección por VIH/Sida en un hijo, siga siendo el mecanismo por el que una mujer conoce de su infección. Para ello, la capacitación y sensibilización del personal de salud, son pilares fundamentales.

3.- Ofrecer servicios de salud con calidad y calidez a las mujeres infectadas por el VIH, para prevenir el desarrollo de neoplasias en especial el cáncer cervicouterino.

4.- Insistir ante las instancias académicas, científicas y organizaciones no gubernamentales y gubernamentales, con trabajo sobre mujeres y VIH/ Sida, la realización de investigación cuantitativa y cualitativa en la población femenina, que permita contar con elementos para realizar acciones más efectivas en la prevención y control de esta epidemia.

5.- Promover en los espacios e instancias, en 


\section{Vera-Gamboa.}

donde la mujer esté desempeñando trabajo remunerado, que las mujeres trabajadoras disfruten de condiciones laborales humanas y dignas y cuenten con información de la repercusión que sobre la salud, familia y sociedad, tiene la epidemia del VIH/Sida y la violencia.

\section{COLOFóN.}

Hoy el gran reto como sociedad es generar diferentes formas de concebir las masculinidades, las femineidades y las sexualidades, así como nuevas y diferentes formas de relacionarnos como hombres y mujeres. También es importante encontrar nuevas maneras de socialización en lo sexual, que incorporen la perspectiva de género y lleven a relaciones equitativas, que permitan reducir la vulnerabilidad femenina al VIH/Sida.

Debemos considerar que la prevención del VIH/ Sida es un compromiso de todos aquellos que conformamos la sociedad. Como hombres y mujeres, profesionales y ciudadanos, desde nuestros diferentes espacios, asumamos nuestro compromiso en la lucha y prevención del VIH/Sida.

\section{REFERENCIAS.}

1.- Pneumocystis pneumonia. Los Angeles. MMWR. Morb Mortal Wkly Rep 1981;30:250-2.

2.- Veinte años de VIH/Sida. Disponible en: http:/ www.unaids.org/fac_sheet/files/AIDStwenty_sp.html. Octubre 2002.

3.- Resumen Mundial de la epidemia del VIH/Sida. Diciembre 2001. Disponible en : http://www.unaids.org. Diciembre 2001.

4.- UNAIDS WHO.AIDS epidemic update: December 2000. Geneva Joint United Nations Programme on HIV/AIDS. Disponible en: http://www.unaids.org. December 2000.

5.- Elias CJ. Prevención del VIH/Sida en comunidades de escasos recursos. Out Look 2001; 19:1-8.

6.- Tasas de incidencia acumulada de Sida, por entidad federativa.Dirección General de Epidemiología. Registro Nacional de Casos de Sida. Datos al 30 de junio de 2002. Centro Nacional para la Prevención y Control del VIH/Sida.
Dirección de Investigación. Subdirección de Investigación e Información Estratégica. Departamento de Análisis y Tendencias.

7.-Servicios de Salud de Yucatán. Departamento de Vigilancia Epidemiologica. Programa de Prevención y Control de VIH/ Sida. Diciembre 2001.

8.- Hartigan P. Enfermedades transmisibles, género y equidad en la salud. Organización Panamericana de la Salud. Publicación ocasional No.7. 2001, p.11.

9.- Gender and HIV/AIDS: taking stock of research and programmes. UNAIDS Best Practice Collection. Marzo 1999.

10.- Rico B, Liguori AL. Mujeres y VIH/Sida: reflexiones sobre la situación actual y algunos retos legislativos. Sida/ ETS 1998; 4:40-7.

11.- Iversen A. Genital shedding in women. AIDS Patient Care. 1999; 13:695-701.

12.- Moscicki A, Holland C, Vermund S. Cervical ectopy in adolescent girls with and without human immunodeficiency virus infection. J Infect Dis 2001; 183:865-70.

13.- Del Rio-Zolezzi A, Liguori AL, Magis-Rodríguez C, Valdespino-Gómez JL, García-García ML, Sepúlveda-Amor J. La epidemia del VIH/Sida y la mujer en México. Salud Pública Mex 1995; 35:581-91.

14.- Uribe ZP. Situación epidemiológica y social del VIH/ Sida en las mujeres y respuestas gubernamentales. Reunión Nacional Mujer y Sida situación actual, investigaciones, acciones y retos para el futuro. México DF. Junio 2001.

15.- Salgado SN, Díaz-Pérez MJ, Factores psicosociales y conducta sexual riesgosa para el Sida en mujeres rurales involucradas en la migración México-Estados Unidos. Salud Reproductiva y Sociedad 1994; 1(3):9-12

16.- Goldzieher SM, Deren S, Shulman LC. Género y cultura como factores de riesgo del VIH/Sida en las mujeres hispanas de los Estados Unidos de América. En:. Rico B, Vandale S, All B, Liguori AL. Ed Situación de las mujeres y el VIH/Sida en América Latina. México 1997. p. 85-100.

17.- Karungari K. Los jóvenes y el Sida. ¿Podemos evitar una catástrofe?. Population Report 2001; XXIX (Serie L): p. 13.

18.- Heise 1, Ellsberg M, Gottemoeller M. Ending Violence against women. Population Report 1999;XXVII(Serie L):p.15.

\section{Revista Biomédica}




\section{Género, violencia y VIH/Sida.}

19.-Vera-Gamboa L, Góngora-Biachi RA, Pavía-Ruz N, LaraPerera D, Alonzo-Salomón L, Pérez-Carrillo H, Gáber-Osorno D. Aceptabilidad a la prueba de anticuerpos al virus de inmunodeficiencia humana (Ac-VIH) en mujeres embarazadas sin seguridad social en Yucatán, México. Enf Infec Microbiol 2000; 20(Suppl):50.

20.- Pedrosa L. Prevención de la transmisión materno-infantil del VIH: algunos resultados y lecciones aprendidas. Reunión Nacional Mujer y Sida situación actual, investigaciones, acciones y retos para el futuro. México DF Junio 2001.

21.- Castro-Sansores C, Pavía-Ruz N. La detección de anticuperpos contra el virus de inmunodeficiencia humana como evaluación prenupcial. Rev Biomed 1995;6:53-4.

22.- Góngora-Biachi RA. La infección de VIH y el Sida en Yucatán: un análisis situacional basado en la investigación. Enf Infec Microbiol 2000; 20:207-13.

23.- Gónzalez Losa MR, Puerto-Solís M, Polanco-Marín GG, Reyes-Ortega A, Puerto FI. Identificación de papiloma virus 6/11, 16/18 y 31/33/51 en cáncer cervicouterino en la ciudad de Mérida, Yucatán, México. Estudio preliminar. Rev Biomed 1996; 7:79-84.

24.- Vera-Gamboa L, Góngora-Biachi R, Castro-Sansores C, González-Martínez P, Váladez-González N, Pavía-Ruz N, et $a l$. Prevalencia de papilomavirus y displasia cervical en un grupo de mujeres con infección por el virus de inmunodeficiencia humana en Yucatán, México. Enf Infec Microbiol 1999; 19(Suppl):S44.

25.- Organización de las Naciones Unidas. Resolución Naciones Unidas 48/104, Diciembre de 1993.

26.- Heise L, Pitanguy J, Germain A. Violence against women.The hidden health burden. 1994. Washington DC: World Bank. Discussion Papers No. 225.

27.- García-Moreno C. Violencia contra la mujer. Género y equidad en la salud. Organización Panamericana de la Salud. Publicación Ocasional no. 6. 2000.

28.- World health Organization. Vice against women. A priority health issue. Geneve. WHO:1997.(WHO/FRH/WHD/ 97.8).

29.- Finkler K. Gender, domestic violence and sickness in México. Soc Sci Med 1997; 45:1147-60.

30.- Valdez-Santiago R. Panorama de la violencia doméstica en México: Antecedentes y perspectivas 1997. Disponible en http://www.laneta.apc.org/cidhal/supl/97/04.html. Mayo 2001.

31.- Klevens J. Violencia física contra la mujer en Santa fe de Bogotá: prevalencia y factores asociados. Rev Panam Salud Pública 2001; 9:78-83.

32.- Sauceda-García J. Violencia en la familia: el maltrato a la mujer. Rev Med IMSS 1995; 33:283-7.

33.- Tocaven R. Instituto de Formación Profesional de la Procuraduría General de Justicia del Distrito Federal.1989.

34.- Hijar Mc, Tapia JR, Lozano R, Chavez R. Violencia y lesiones. Salud Mental 1992; 15:15.

35.- Ramos-Lira L, Saltijeral-Méndez MT, Romero-Mendoza M, Caballero-Gutiérrez MA, Martínez-Vélez NA. Violencia sexual y problemas asociados en una muestra de usuarias de un centro de salud. Salud Pública Mex 2001; 43:182-91.

36.- Oliva-Peña Y. Violencia contra la mujer en la relación de pareja. XV Jornadas Académicas de la Facultad de Medicina. Universidad Autónoma de Yucatán. Mérida, Yucatán, Marzo 2001.

37.- García-Ramírez M. Violencia intrafamiliar. En Violencia intrafamiliar, violencia de género. México: Fundación Mc Arthur; 1995. p. 232.

38.- Walker A. Violencia de género en : Violencia intrafamiliar, violencia de género. México: Fundación Mc Arthur; 1995. p. 232.

39.- Granados-Shiroma M. Salud reproductiva y violencia contra la mujer. En : Los silencios de la salud reproductiva, violencia, sexualidad y derechos reproductivos. México: Fundación Mc Arthur; 1998. p. 76.

40.- Góngora-Biachi RA, González-Martínez P. Anticuerpos contra el Virus de Inmunodeficiencia Humana (VIH) en una población de prostitutas de Mérida, Yucatán, México. Rev Invest Clin 1987; 39:305-6.

41.- Góngora-Biachi RA, Pavía-Ruz N, González-Martínez P, Puerto-Manzano F. Female prostitution and HIV infection in Yucatán, México. Fourth RCMI International AIDS Symposium. San Juan, Puerto Rico. November 1994 [abstract p.56].

42.- Estébañez P, Fitch K, Najera R. HIV and female sex workers. Bol Of Sanit Panam 1993; 115:415-37.

Vol.14/No.4/Octubre-Diciembre, 2003 


\section{Vera-Gamboa.}

43.- Hernández AM, Uribe ZP. Diversity in commercial sex work systems: preliminary findings from Mexico City and their implications for AIDS interventions. In: Chen LC, Sepúlveda J, Segal ST,ed. AIDS and Women's Reproductive Health. New York: Plenum Press; 1991. p.179-94.

44.- Uribe SF, Hernández AM, Conde JC, Juárez FL, Alen B, Anaya OR, Del Río CHC, Uribe ZP, Zaldoundo B. Low prevalences of HIV infection and sexually transmitted disease among female commercial sex workers in México city. Am J Public Health 1997; 87:1012-5.

45.-Peralta-Sánchez A, Hernández-Hernández R, Estudio integral de la prostitución en el municipio de Tehuacán, Puebla. Una visión íntima. Archivos Hispanoamericanos de Sexología 2001;VII:31-63.

46.- Góngora-Biachi RA, Pavía-Ruz N, Vera-Gamboa L, Moguel-Rodriguez W, González-Martínez P, FloresAbuxapqui J, Lara-Perera D, Alonzo-Salomón L,. Knowledge, behavior, and seroprevalence towards HIV infection among female sexual workers. Rev Biomed 2002; 13:257-63.

47.- Ford N, Koetsawang S. A pragmatic intervention to promote condom use by female sex workers in Thailand. Bull WHO 1999; 77: 888-893.

48.- Novelo-Del Valle A, Quintal-Duarte R, Toledo-González J, Vera-Gamboa L. Nivel de autoestima en un grupo de trabajadoras sexuales en Yucatán, México y su impacto en la salud. XXII Asamblea Nacional de Médicos Generales y Familiares. Acapulco, Gro., México. Mayo 2002. 